\title{
Arteriotomy for Carotid Endarterectomy, from Common to Interna or Externa?
}

\author{
Süreyye Talay ${ }^{1}$, [MD] \\ ORCID: 0000-0002-4908-3850
}

${ }^{1} 29$ Mayıs States Hospital Department of Cardiovascular Surgery, 06100, Dikmen, Ankara, Turkey
Corresponding Author:Süreyya TALAY 29 Mayıs States Hospital Department of Cardiovascular Surgery, 06100, Dikmen, Ankara, Turkey e-mail: suereyyatalay@gmail.com

\section{nee) A B S TRACT Cer}

Objective: Carotid endarterectomy is an evolving and changing procedure over its almost 70 years history. Surgery is commonly described as a total removal of plaques from the carotid tree maintained via an arteriotomy from common carotid artery to internal carotid artery. We aim to express our opinion that an incision to external carotid artery may always provide adequate reach to a plaque of internal carotid artery side. So, there is no need of internal carotid artery involvement during arteriotomy. Materials and Methods: 73 patients were included to study. All patients underwent single-sided Carotid Endarterectomy. Group A ( $n=61)$ and Group B ( $n=12)$. Group A included our primary cases Carotid Endarterectomy with artery incision Common Carotid Artery to External Carotid Artery in 61 patients. Group B patients of 12 cases received an artery incision from Common Carotid Artery to Internal Carotid Artery. Results: 2 patients in Group A (3.2\%) and 2 patients (16.6\%) in Group B died at the early postoperative period due to severe neurological deteriorations in $3(4.1 \%)$ and myocardial infarction in 1 (1.3\%) case, $\mathrm{p}<0.05$ (SS, statistically significant). Overall mortality was 4 cases by $5.4 \%$. None of these patients were presenting contralateral serious carotid artery lesions. 4 patients (6.5\%) from Group A and 3 patients (25\%) from Group B were receiving haemodialysis, perioperatively $\mathrm{p}<0.05$ (SS, statistically significant). Transcient minor neurological complications such as lingual deviation and/or facial asymmetry which were associated with N.Hypoglossus injuries, was observed in 8 cases and 2 cases in Group A (10.9\%) and Group B (16.6\%), respectively ( $p>0.05$ statistically non-significant)

Conclusion: By this Common Carotid Artery to External Carotid Artery running arteriotomy, it is possible to avoid intraoperative Internal Carotid Artery narrowing and endothelial deterioration which may cause early and/or late intraluminal thrombosis tendency. For carotid endarterectomy arteriotomy, we always prefer common to externa.

Key words: Carotid endarterectomy, artery incision, surgical modification

Received: 14 July 2020, Accepted: 15 July 2020,

Published online: 30 September 2020

\section{INTRODUCTION}

Carotid endarterectomy (CEA) is an evolving and to remove these plaques primarily from internal ICA. changing procedure over its almost 70 years histo- Conditions of external carotid artery (ECA) luminalry. CEA is commonly described as a total removal of ly are sometimes ignored during surgery, at least in plaques from the carotid tree maintained via an ar- several cases [1]. An incision of ICA causes a reasonteriotomy from common carotid artery (CCA) to in- able narrowing of lumen and a certain endothelial ternal carotid artery (ICA). Generally, surgical aim is deterioration which may result with a postoperative 
thrombosis and/or re-stenosis. In our surgical modification, we applied our arteriotomy incision from CCA to ECA. ICA was kept free of incision. All cases were operated due to severe internal artery stenosis with or without common and/or external artery stenosis. We also tried to avoid shunt application intentionally, in all cases. Shunt was applied when it was completely necessary. We aim to express our opinion that an incision to ECA may always provide adequate reach to a plaque of ICA side. So, there is no need of ICA involvement during arteriotomy.

\section{MATERIALS AND METHODS}

\section{Data Collection}

Data was accumulated from two different medical centers between the dates of March 2016 to August 2018. This study was peformed retrospectively. Study was approved by hospital administrations. 73 patients were included to study. Co-morbidities which required simultaneous surgeries such as coronary artery disease and cardiac valve pathologies, severe artery stenosis in different locations in extremities and percutaneous interventions were accepted as exclusion criteria. All patients underwent single-sided CEA.

\section{Surgical Procedures}

Premedication performed with Propofol $3-4 \mathrm{mg} / \mathrm{kg}$, Fentanyl Citrate $10 \mathrm{microgr} / \mathrm{kg}$ and Pancuronium bromid 0,1-0.2 mg/kg. Anaesthesia continued with additional doses of Propofol 2-5 mg/kg/h. Our choice of inhaled anaesthesia was Sevoflurane.

We applied different CEA techniques in two different group of patients ( $n=73)$ : Group $A(n=61)$ and Group $B(n=12)$. Group $A$ included our primary CEA cases with artery incision CCA to ECA in 61 patients.
Group B patients of 12 cases received an artery incision from CCA to ICA. All operations were performed under general anasthesia. Initial skin incision medial to the sternocleidomastoid muscle was used to reach the carotid system. The latter was completely dissected free from surrounding tissue. 15000 IU of heparin was routinely administered. Vascular clamps were applied to CCA, ECA and ICA after silastic vascular loops. A longitudinal arteriotomy medially to CCA was made and extended to ECA in Group A ( $n=61$ ) (Figure 1) and ICA in Group B ( $n=12)$. Atherosclerotic plaque was primarily freed from ECA and than from ICA. Finally, the plaque was cut free totally in the CCA by blunt dissection (Figure 2). At this stage, debris removal achieved by saline irrigation. Afterwards, arteriotomy was closed primarily by using $6 / 0$ polypropylene sutures when possible. Carotid shunts ( $6 \mathrm{f}, 20 \mathrm{~cm}$ in lenght) from CCA to ICA $(n=5)$ and carotid patchplasty $(n=5)$ were applied when surgical situation left us no other choice.

\section{Statistical Analysis}

The data are expressed as proportions or as the mean \pm standard deviation. Differences in continuous variables were analyzed by Student t tests. Results were considered significant if $p$ values were less than $<0.05$.

\section{RESULTS}

\section{Patient Characteristics}

Study perioperative data was accumulated from two different surgery centers. Preoperatively, a routine dupplex ultrasonography and a carotid system computerised tomography were performed in all cases. Preoperative data are summarized in Table 1. 
Table 1. Preoperative parameters

\begin{tabular}{|c|c|c|c|}
\hline Variable & Group A(n=61) & Group $B(n=12)$ & $P$ value \\
\hline CEA & 61 & 12 & \\
\hline Mean age & $63.7 \pm 2.2$ & $61.2 \pm 4.4$ & $p>0.05$ \\
\hline Male & 40 (65.5\%) & $8(66.6 \%)$ & $p>0.05$ \\
\hline $\mathrm{BMI} \mathrm{kg} / \mathrm{m}^{2}$ & $2988 \pm 1.8$ & $3001 \pm 0.6$ & $p>0.05$ \\
\hline Stroke history & $21(34.4 \%)$ & $4(33.3 \%)$ & $p>0.05$ \\
\hline COPD & $10(16.3 \%)$ & $3(25 \%)$ & $\mathrm{p}<0.05(\mathrm{SS})$ \\
\hline DM & $10(33.3 \%)$ & $4(33.3 \%)$ & $\mathrm{p}<0.05(\mathrm{SS})$ \\
\hline Dialysis & $4(6.5 \%)$ & $3(25 \%)$ & $\mathrm{p}<0.05$ (SS) \\
\hline CABG hisory & $17(11 \%)$ & $2(16 \%)$ & $p>0.05$ \\
\hline
\end{tabular}

Values presented are mean \pm SD. BMI: Body mass index, CABG: Coronary artery bypass grafting, COPD: Chronic obstructive pulmonary disease, DM: Diabetes Mellitus, SS: Statistically significant.

Two patients in Group A (3.2\%) and 2 patients (16.6\%) in Group B died at the early postoperative period due to severe neurological deteriorations in $3(4.1 \%)$ and myocardial infarction in 1 (1.3\%) case, $\mathrm{p}<0.05$ (SS, statistically significant). Overall mortality was 4 cases by $5.4 \%$. None of these patients were presenting contralateral serious carotid artery lesions. 4 patients (6.5\%) from Group A and 3 patients (25\%) from Group B were receiving haemodialysis, perioperatively $\mathrm{p}<0.05$ (SS, statistically significant). Transcient minor neurological complications such as lingual deviation and/ or facial asymmetry which were associated with
N.Hypoglossus injuries, was observed in 8 cases and 2 cases in Group A (10.9\%) and Group B (16.6\%), respectively ( $p>0.05$ statistically non-significant) . Patchplasty was necessary in 5 from Group A (8.1\%). No patchplasty was applied in Group B. We tried to avoid carotid shunt from common to interna in all cases. However, carotid shunt was mandatory in 4 patients and in 1 patient from Group A (6.5\%) and B (8.3\%), p<0.05(SS) .

\section{Operative Data}

All surgeries were performed in a similar fashion. Table 2 depicts our intraoperative data in both Groups.

Table 2. Operative data

\begin{tabular}{|c|c|c|c|}
\hline Variable & Group $A(n=61)$ & Group $B(n=12)$ & $P$ value \\
\hline CEA & & 12 & \\
\hline Operation (minute) & $61.5 \pm 30.1$ & $67.1 \pm 11.9$ & $p>0.05$ \\
\hline CCT (minute) & $23.4 \pm 6.9$ & $21.8 \pm 1.9$ & $p>0.05$ \\
\hline $\mathrm{R}$ - side & 40 (65.5\%) & $7(58.3 \%)$ & $p>0.05$ \\
\hline Shunt & $4(6.5 \%)$ & $1(8.3)$ & $p>0.05$ \\
\hline Patchplasty & $5(8.1 \%)$ & 0 & $\mathrm{p}<0.05$ (SS) \\
\hline Intubation (minute) & $440 \pm 38.8$ & $456 \pm 9.4$ & $p>0.05$ \\
\hline ICU(day) & $3.1 \pm 4.2$ & $2.7 \pm 2.1$ & $p>0.05$ \\
\hline Hospitalization (day) & $6 \pm 1.6$ & $5.8 \pm 0.4$ & $p>0.05$ \\
\hline
\end{tabular}

Values presented are mean \pm SD, CCT: Cross clamp time, R-side: Right side, CEA: Carotid endarterectomy, ICU: Intensive care unit, SS: Statistically significant.

In overall results, we observed statistically significance $(p<0.05)$ only in patchplasty parameter between Group A and Group B. All other operative data were similar. Reoperations were performed due to wound hematoma were mandatory in 4 patients in Group A (6.4\%) and 2 patients in Group B (8.3\%). In this parameter, there was not a statistically significant difference ( $p>0.05) .3$ of these patients from Group A were shunt applied patients. 


\section{DISCUSSION}

In a standart CEA surgery, after cervical incision parallel and anterior to sternocleidomastoid muscle, we visualize internal jugular vein. We almost always ligate common facial vein. Dissection is continued proximally to the anterior CCA to ensure vagus nerve safety. Primary attention is essential for glossopharengeal-vagus-hypoglossal and marginal mandibular branch of facial nerves. We mobilize and turn CCA with vascular tapes prior to ECA and ICA. 'No touch to hypoglossal nerve' is important for us during distal retractions for surgical exposure, if possible. Excessive and prolonged retractions are prone to temporary neurological complications such as lingual deviations and facial asymmetries [2]. In our study group, 8 patients from Group A and 2 patients group B demonstrated the minör neurological complications. There were no statiscally significant difference for this parameter. Thus, an ECA extending incision seems not to elevate neurological complications in our study. In higher located carotid bifurcations, different surgical maneuvers are suggested in several publications [3] such as; complete sternocleidomastoid mobilization distaly to its insertion, dividing of digastric muscle and styloid process transection with mandible subluxation. In our cases, we did not apply any of these maneuvers above. Furthermore, for an easier removal of plaque, we prefer to mobilize ICA to a point where this artery is completely intact.

At this point, in aim to avoid a reflex sympathetic bradycardiae applied 3-5 mL of lidocaine topically. After 5000 U Heparin IV and clamping of ICA-CCAECA, standart CEA surgery is described as starting from CCA to ICA [4]. In our study, Group A arteriotomy is continued to ECA as shown in Figure 1. In Group $B$, incision continued to ICA, untill the artery is free of plaques. CEA performed primarily by dissecting the plaque sharply at its proximal portion in CCA. Than the removal continued with an elevator to ECA and finaly to ICA, conventional [5]. Back-flow of ICA is always controlled to ensure the procedure and to removal debris from ICA region. In Group A, we were able to remove plaque totally in all 61 cases. Despite the fact that authors like Schnaudigel et all [6] reported an ICA extention to be beneficial for total removal, we believe in the contrary. An ECA incision is almost always adequate for a total ICA plaque removal (Figure 2). Thus, it is possible to maintain ICA endothelial integrity by avoiding an ICA arteriotomy. Furthermore, an ICA narrowing by arteriotomy closure also is eliminated by this ECA extending incision.

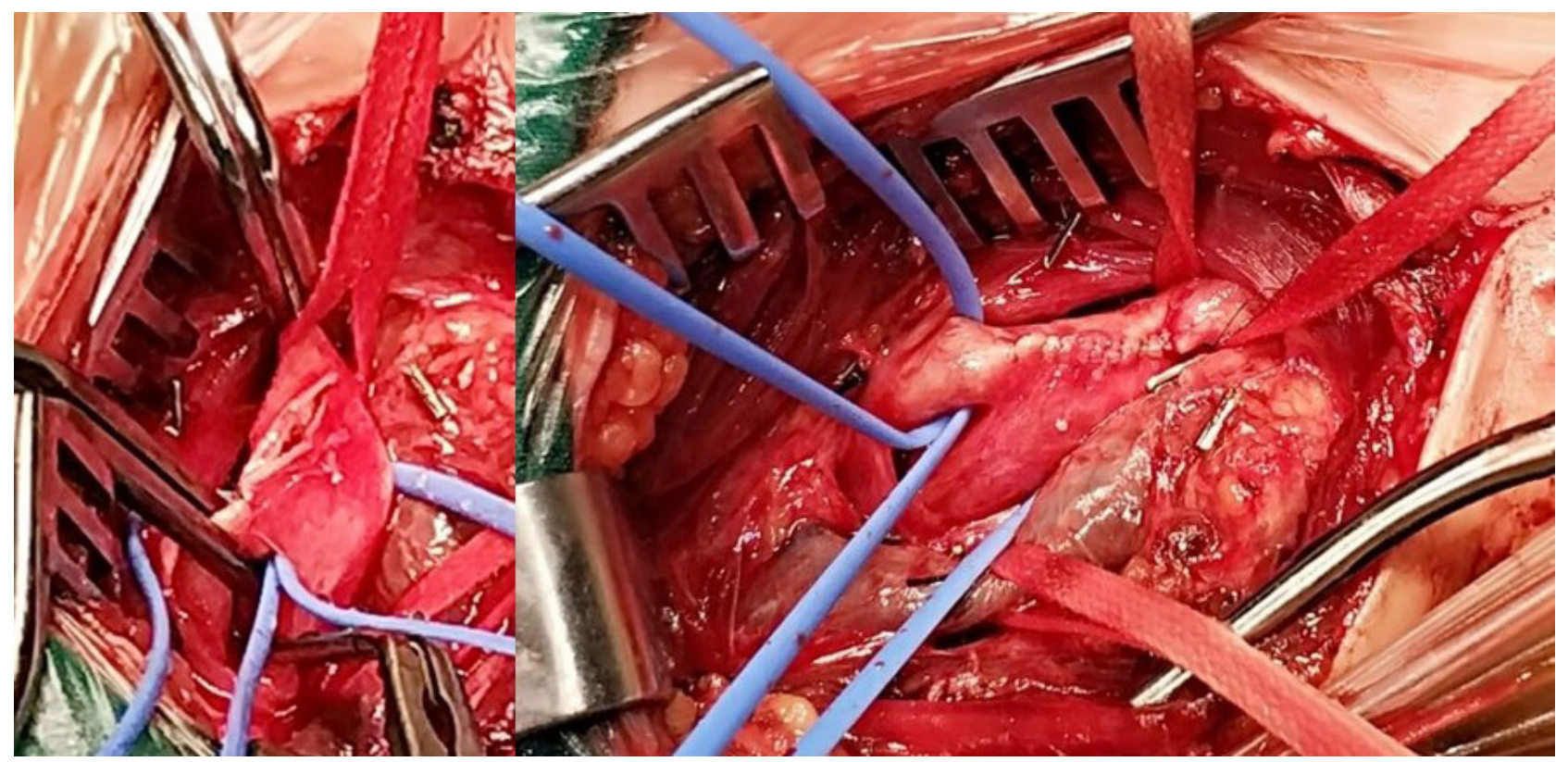

Figure 1. Carotid arteriotomy from common to externa 


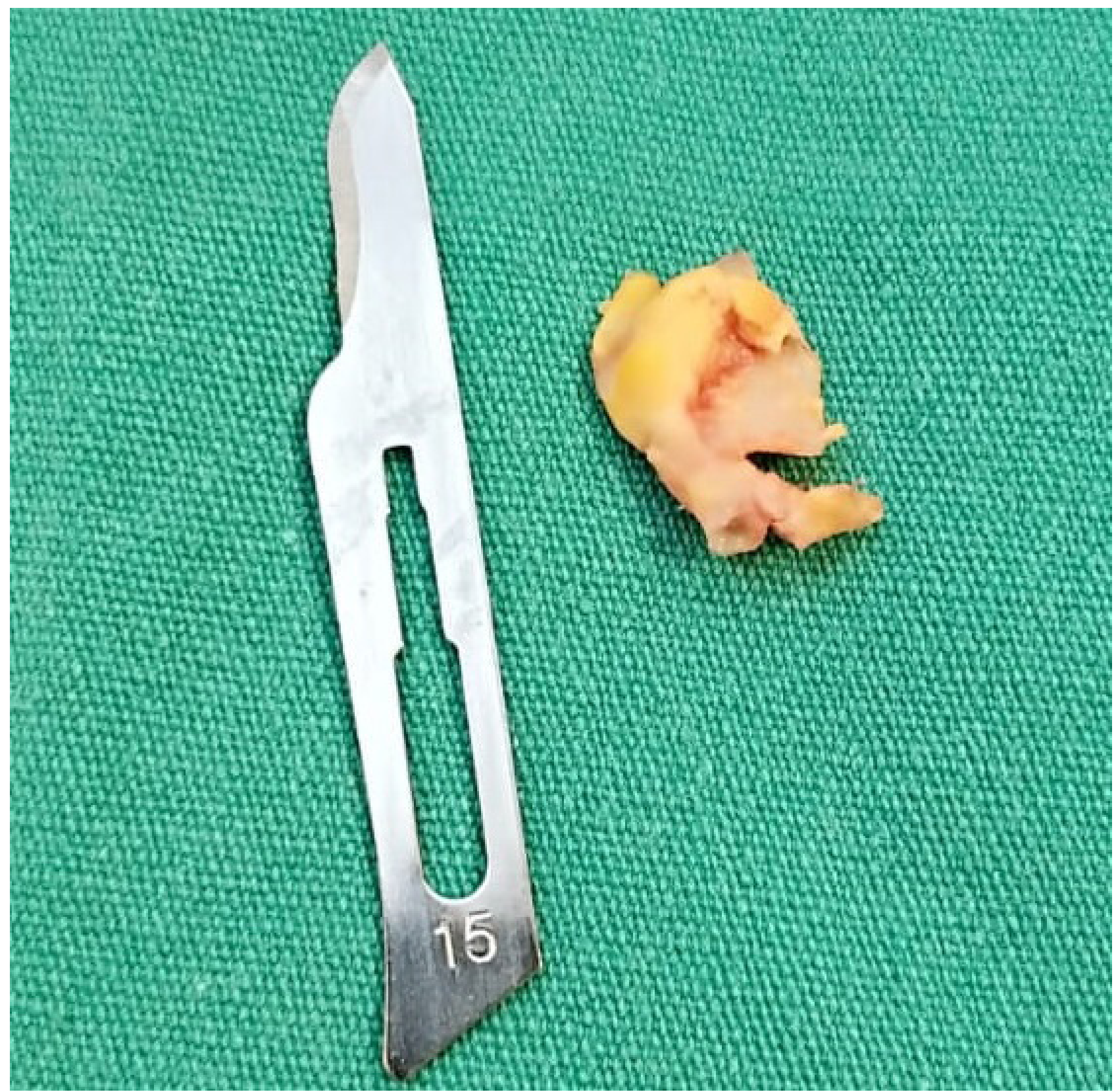

Figure 2. Succesfully excised carotid internal plaque

During closure of arteriotomy, we used patchplasty in 5 patients from Group A. In which, 3 autogenous saphenous vein and 2 Dacron grafts were used. There was a statistical significantly difference when compared to Group A where no patchplasty was necessary. In larger series, we believe this difference to lower.

Early postoperative mortality rate was $5.4 \%$ by 4 cases in our study, 2 patients from each group. Elaboration of mortality presented a percentage $3,2 \%$ in Group A and \%16.6 from Group B. This data presented a statistically difference between groups $(p<0.05)$. However, we believe that this latter finding may need enlightenment by larger series of CEA. As shown in Table 1, Group B presented that there were more patients by means of co-morbidities such as COPD $(n=3,25 \%)$, dialysis $(n=3,25 \%)$ and CABG history $(n=2,16 \%)$. This different rates of co-morbidities may explain the higher tendency in mortality of Group B. Our mortality rates presented a similarity when compared to literature [7] with larger series. As in our findings, Pujol et al also report that presence of DM, COPD and prior severe vascular pathologies are the risk factors of mortality for CEA.

Main topic of our opinion depends on the question; 'Arteriotomy for Carotid Endarterectomy, from Common to Interna or Externa? '. During our 
experience of carotid endarterectomy, common to externa is a better decision in and for all cases. However, the latter choice is not possible in each case as we performed common to interna incisions in 12 cases from Group B. Simply because it was not possible to maintain the internal artery plaque removal from an external artery continuing arteriotomy in these cases. In situations such as; a really narrow ECA where an arteriotomy is not possible, totally calsified ECA which blocks an incision, aberrant orific and more proximally arising ECA as in an anatomical variation and accidental total rupture of ECA during surgery with a total seperation of ECA branch from the carotid tree, we were obliged to perform an ICA arteriotomy. In 1 case from our Group B, we encountered a dense and thick adhesion which probably caused by a local infection or inflammation over and around ECA region, we were not able to dissect ECA and turn this artery in order to further the arteriotomy incison towards it. In this patient, a common to ICA arteriotomy also was mandatory. Other than these situations listed above, our primary objective was to run the arteriotomy from common to externa.

A recent study from Savardekar et al [8] underline the timing of CEA; as they reported that traditional view towards carotid revascularization was that intervention was to be delayed at least 6 weeks after acute stroke, as studies published in 1960s and 1970 s noted that early surgical intervention after an ischemic stroke was associated with a high rate of postoperative cerebral hemorrhage. Over the turn of the 20th century, there was a trend towards early surgery for carotid artery stenosis after several studies showed the high risk (range, $9 \%-12 \%$ ) of having a recurrent stroke in the intervening 6 weeks wait period. With improved surgical techniques and better peri-procedural critical care, several centers started undertaking early CEA and demonstrated good outcomes. In our opinion, an arteriotomy running to ECA without an involvement of ICA, surgery is safer by means of a lesser possibilty for early thrombosis in ICA area, postoperative dissection and total occlusion of ICA also is lower and early postoperative bleeding and risk of reoperation also would be less possible due to an absence of ICA incision. Thus, we prefered a 4 weeks of waiting period after cerebrovasculary event and/or stroke when possible. Because, parallel to Savardekar's report we also believe that a strategy of prolonged intensive medical therapy, in the presence of severe carotid artery stenosis and a stroke history, may play a role in alleviating the high risk of very early recurrent stroke.

\section{Limitations of The Study}

This retrospective manuscript describes a surgical modification for CEA; an incision from common to external carotid artery. We are well aware that this modification is not a major differentiation. Furhermore, patient number also are just adequate to have a common opinion. Larger series may present more valuable statistical results but we do not believe that would make a difference in statistical analysis compared to ours. Furthermore, the difference in numbers of cases between Group $A$ and Group B is large, 61 to 12 . We accept that this numerical difference may lead to statistically misleading results. We are collecting long-term results and re-occlusions of our cases to report in the future.

\section{CONAUSION}

In CEA, artery incision from CCA to ECA always presents a perfect exposure of ICA internal anatomy. Thus, we can perform ICA plaque removal via this modification. In 61 operations by this ECA incision, surgery was as 'comfortable' as an ICA extended incision for endarterectomy. By this CCA to ECA running arteriotomy, it is possible to avoid intraoperative ICA narrowing and endothelial deterioration which may cause early and/or late intraluminal thrombosis tendency. For carotid endarterectomy arteriotomy, we always prefer common to externa.

\section{CONFUCT Of INTEREST STATEMENT}

We do not have any conflict of interest with any third parties and/or organizations. 


\section{reo REFERENCES Con}

[1] Landis GS, Faries PL. A critical look at "high-risk" in choosing the proper intervention for patients with carotid bifurcation disease. Semin Vasc Surg. 2007; 20 (4):199-204.

[2] Qin F, Sun Y, Hu W, et al. The relationship between preoperative serum cortisol level and the stability of plaque in carotid artery stenosis patients undergoing carotid endarterectomy. J Thorac Dis. 2016; 8 (7):1611-7.

[3] Lal BK, Beach KW, Roubin GS et al. Restenosis after carotid artery stenting and endarterectomy: a secondary analysis of CREST, a randomised controlled trial. Lancet Neurol. 2012; 11(9):755-63.

[4] Hye RJ, Mackey A, Hill MD et al. Incidence, outcomes, and effect on quality of life of cranial nerve injury in the Carotid Revascularization Endarterectomy versus Stenting Trial. J Vasc Surg. 2015; 61 (5):1208-14.
[5] Tedesco MM, Lee JT, Dalman RL et al. Postprocedural microembolic events following carotid surgery and carotid angioplasty and stenting. J Vasc Surg 2007;46:244-50.

[6] Schnaudigel S, Groschel K, Pilgram SM et al. New brain lesions after carotid stenting versus carotid endarterectomy: a systematic review of the literature. Stroke 2008; 39:1911-9.

[7] Pujol Lereis VA, Ameriso S, Povedano GP et al. Morbidity and mortality of carotid endarterectomy. Medicina ( $B$ Aires). 2011;71(5):449-53.

[8] Savardekar AR, Narayan V, Patra DP, et al. Timing of Carotid Endarterectomy for Symptomatic Carotid Stenosis: A Snapshot of Current Trends and Systematic Review of Literature on Changing Paradigm towards Early Surgery. Neurosurgery. 2019; 85(2):214-225. 